\title{
Research on Interactive Soundscape Design for Urban Landscape
}

\author{
Li Xiaohong ${ }^{1,}$, Wang Jinxiao ${ }^{1, \mathrm{~b}}$, Zhu Hongxuan ${ }^{1, \mathrm{c}}$ \\ ${ }^{1}$ Qingdao University of Technology Department of art and design Qingdao, Shandong
}

\begin{abstract}
This paper intends to discuss the concept and design elements and methods of the interactive soundscape for urban landscape through the analysis on the influence relationship of components of a soundscape for urban landscape. With the analysis on the composition of the acoustic environment for urban landscape as the starting point, it finds out the implicit relationship of components of the acoustic environment, introduces the concept of interactive design, proposes the concept and components of the interactive soundscape for urban landscape and discusses its design methods and specific process with the case simulation method, taking the soundscape design of landscape nodes in Zhongshan Park, Qingdao as an example.
\end{abstract}

\section{Introduction}

With the continuous development of social economy, the demand of people for urban landscape experience is no longer limited to visual effects, but also involves multisensory experience. Hearing, as an important method of information perception, has received a lot of attention. The concept of "soundscape for landscape" thus emerged in the research in relevant fields.

The concept of "soundscape for landscape" is essentially the introduction and expansion of the concept of "soundscape" in the field of landscape architecture. The concept of "soundscape" originated in the 1960s, which was defined by Canadian musicologist R. Murray Schafer as an acoustic environment with emphasis on individual or social perception and understanding modes ${ }^{[10]}$. "Soundscape for landscape" has been defined by the International Standards Organization as an acoustic environment in a given scene perceived by individuals, groups or communities ${ }^{[1]}$ with emphasis on the close relationship between human perception and the environment.

The research on the soundscape for urban landscape has shown certain development in recent years with the rising attention to the quality of urban human settlements in related fields, mainly including the definition of the concept of soundscape in the broad sense and the exploration of methodology ${ }^{[2]}$, research on soundscape quality evaluation based on human needs ${ }^{[4,7,8]}$, the construction of soundscape infrastructure ${ }^{[3]}$ and research on cultural and aesthetic thoughts of garden soundscape construction $^{\left[5^{\sim} 6\right]}$, etc. Most of the researches focus more on the impact of environmental sounds on people in the soundscape and are lacking in the attention to the active participation of human voice in the soundscape. The experience of soundscape for urban landscape requires further research. Therefore, it is necessary to combine the joint participation of environmental sound and human voice in the soundscape to create landscape objects that can dynamically reflect sound changes and realize the real-time interaction between human voice and environmental sound, which is positive for users to obtain a better soundscape experience.

\section{Research on interaction of soundscape for urban landscape}

\subsection{Soundscape composition and internal relations}

The soundscape for urban landscape is mainly composed of sounds, listeners and the environment. Sounds, as the basis of soundscape, generate a soundscape atmosphere through itself and the objective physical landscape space and produce a certain impact on listeners in the environment. Listeners are main participants of soundscape. The quality of soundscape experience is closely related to physical, psychological and social characteristics of listeners. Meanwhile, listeners have the creative ability to change the soundscape. The environment is an objective carrier of soundscape. Spatial attributes of the landscape space, the sound transmission medium and other physical elements have an important effect on the sound transmission quality.

In the soundscape for urban landscape, sounds can be divided into keynote sounds, signal sounds and sign sounds ${ }^{[11]}$ (Table 1) based on the difference of sounds and physical properties of the landscape space:

- Keynote sounds: Keynote sounds of the natural type are related to basic elements of nature in the environment itself. For example, the sound of rain often causes soothing and relaxed emotions, and certain types of birdsongs can bring about pleasure and enterprise. Keynote sounds of social production activities are often associated with planning, location and other uncontrollable factors. For example, the noisy whistle in adjacent street areas and the

a1xh.802@163.com
b1634099182@qq.com

c276744125@qqcom 
sound of machine maintenance in the park often make people feel agitated.

- Signal sounds: The emotional effect of artificial sounds made for management purposes such as safety and order maintenance largely depends on the setting of sound quality.

- Sign sounds: Such sounds are highly recognizable and can reflect the unique culture and other characteristics of the region. For example, the sound of waterfalls and fountains can make tourists positive, and beautiful background music drives the emotional experience of the place.

Table 1 Sound classification of soundscape for urban landscape (Revised according to the summary of literature ${ }^{[11]}$ )

\begin{tabular}{|c|c|c|}
\hline $\begin{array}{l}\text { Sound } \\
\text { category }\end{array}$ & Interpretation & Example \\
\hline $\begin{array}{l}\text { Keynote } \\
\text { sounds }\end{array}$ & $\begin{array}{l}\text { Similar to the concept } \\
\text { of background sound, } \\
\text { such sounds are basic } \\
\text { sound characteristics } \\
\text { inherent in the space } \\
\text { and play an important } \\
\text { role in the entire } \\
\text { soundscape } \\
\text { atmosphere. For } \\
\text { example, there are } \\
\text { birdsongs in the forest. }\end{array}$ & $\begin{array}{l}\text { Sound of wind, } \\
\text { sound of rain, } \\
\text { animal sound, } \\
\text { plant sound under wind, } \\
\text { sound of water, } \\
\text { Birdsong, } \\
\text { spatial sound, } \\
\text { and urban noise }\end{array}$ \\
\hline $\begin{array}{l}\text { Signal } \\
\text { sounds }\end{array}$ & $\begin{array}{l}\text { Such sounds have } \\
\text { certain indication } \\
\text { functions, such as the } \\
\text { alarm sound and } \\
\text { ringtone. }\end{array}$ & $\begin{array}{l}\text { Alarm sound, } \\
\text { bell } \\
\text { and ringtone }\end{array}$ \\
\hline Sign sounds & $\begin{array}{l}\text { Sounds that reflect } \\
\text { regional characteristics, } \\
\text { such as highly } \\
\text { recognizable natural } \\
\text { sounds and specific } \\
\text { artificial sounds. }\end{array}$ & $\begin{array}{c}\text { Sounds of peculiar } \\
\text { natural elements } \\
\text {, such as waterfalls, } \\
\text { and artificial ambient } \\
\text { sounds } \\
\text { such as broadcast music. }\end{array}$ \\
\hline
\end{tabular}

According to the analysis on physical characteristics and emotional effects of the soundscape for urban landscape, sounds, listeners and the environment form a complex relationship of interaction (Figure 1). In terms of physical characteristics, the loudness, tone and timbre of sounds contribute to the generation of different sound types and further enable listeners to have different soundscape experience. The population density, age, gender and other physiological factors in the soundscape space have a negative effect on the soundscape, while physical elements of the objective environment of soundscape are crucial to the sound transmission medium. In terms of emotional effects, keynote, signal and sign sounds of the soundscape for landscape meet the emotional experience needs of different people with their respective emotional effects. In addition, research has shown that besides the sound itself, the sound perception of people in the environment is also affected by visual perception $^{[14]}$. Therefore, visual effects of the landscape space environment also play an important role in the auditory experience of people, and emotional elements such as the listener's psychological state also affect the emotional experience of different people for the soundscape for landscape.

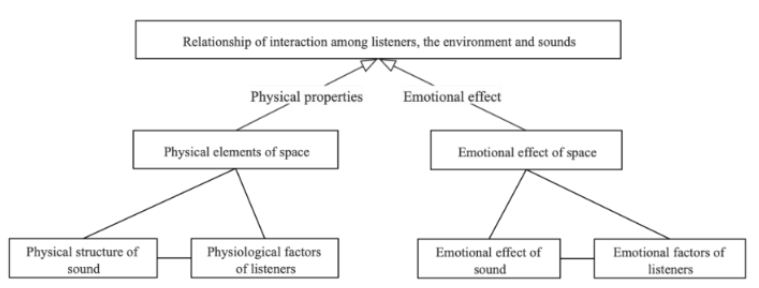

Figure 1 Relation of soundscape components (compiled by the author)

\subsection{Concept of interactive soundscape}

The concept of interactive design originated in the 1980s, which was defined by Alan Cooper as the behavior of artifacts, environments and systems as well as the design and definition of shape elements that convey the behavior $^{[12]}$. Characteristics of interactive design are manifested as active interaction and passive interaction in practical applications, in which the mutual feedback relationship between users and design results is emphasized.

The mutual effect of sounds, the environment and listeners has laid an objective foundation for the discussion on interactive design of the soundscape of urban landscape based on the above discussion on the relationship of its components ${ }^{[9]}$. According to Figure 1, listeners can not only passively receive the function and effect of sounds and the environment in the soundscape, but also participate in the transformation and construction of the soundscape for urban landscape through their own subjective initiative, which reflects the passive experience and active participation of people in the soundscape and shows high consistency with basic features of interactive design.

The interactive soundscape for urban landscape, different from the traditionally recognized soundscape for landscape, puts more emphasis on the active participation of people and the feedback of the landscape to the participation of human voice and realizes the interaction between people and sounds in the form of an interactive landscape carrier. The initiative of interaction is dominated by human behaviors. Listeners realize two-way communication with the landscape through the active input of sounds or the transmission of information, for example, under the speech recognition technology. The passiveness of interaction refers to implicit information transmission, which means that participants receive environmental information passively in the landscape space environment and have emotional or behavioral dialogues with the place.

\subsection{Design elements of interactive soundscape}

From the perspective of interactive design, design elements of the interactive soundscape for urban landscape mainly include interaction participants and media - sounds and interactive devices. The content of sound design mainly includes sound composition and collocation, that is, the arrangement and planning of keynote, signal and sign sounds in the soundscape of a place. Interactive devices of sound are landscape devices 
of sound processing based on modern digital media technology.

With respect to the content of sound design, reasonable artificial sound elements should be incorporated through the design plan based on the summarization and selection of original sounds in the place, and the participation of artificial sounds in the soundscape should be achieved through interactive devices. In the interactive soundscape, sounds enter the control terminal by means of the sensing ability of the input terminal, and the control terminal generates feedback to the original sound in various ways at the output terminal through the control and transformation of information. The technical route for the implementation of interactive devices should pass through three parts - input, control and output terminals ${ }^{[13]}$. The input terminal reflects the external sound perception function of devices, e.g. a microphone. The control terminal refers to the central information control. The information sensed by the input terminal is transmitted to the outside world with the expected effect via the output terminal according to a series of algorithm and parameter settings made by designers and developers. In terms of the form, landscape serves as an objective carrier of the output terminal. The feedback effect of the landscape to sounds should be achieved through multi-sensory effects such as visual, auditory and tactile effects based on specific circumstances of the design plan.

\section{Design methods of interactive soundscape for urban landscape}

\subsection{Overview of design methods}

Based on the discussion of design elements above, designers should, first, measure the original initial acoustic environment of the place and conduct a hierarchical analysis on keynote, signal and sign sounds inherent in the environment; second, get aware of voice characteristics and needs of people in the place through an investigation on them and then plan the level of the feedback acoustic environment after processing; and finally realize the transformation from the initial acoustic environment to the feedback acoustic environment by technical means in the actual design process. Taking the "Little West Lake" landscape node in Zhongshan Park, Qingdao for an example, this paper discusses design methods of the soundscape for urban landscape at all stages successively with the case simulation method.

\subsection{Site investigation stage}

The author, first, chose the tour route along the "Little West Lake" node in Zhongshan Park as a design example; second, modified the sound category based on the sound classification in Table 1 and the actual situation of the site, made a questionnaire with the five-point Likert scale method (1: very dissatisfied, 2 : relatively unsatisfactory, 3 : just so so, 4: relatively satisfied, 5: very satisfied) and invited site users for scoring and evaluation of various sounds; and finally determined the relationship of composition of the initial acoustic environment of the site according to the evaluation result. A total of 55 questionnaires were issued in the investigation and 55 were effectively collected. The survey analysis shows that users of the landscape node are mainly young and middleaged groups at a balanced ratio of men and women (Figure 2 ), indicating that the sound receiving capability of site users is at the average level of the human body. According to the average score of sounds in Figure 4, residents generally believe that natural sounds of the park such as the sound of water, wind and leaves have better effects (Figure 3). The Pearson correlation analysis of sound scores (Table 2 and Figure 4) shows that the sound of water in the place has the highest average correlation coefficient, so it has the most significant effect on the soundscape experience of users.

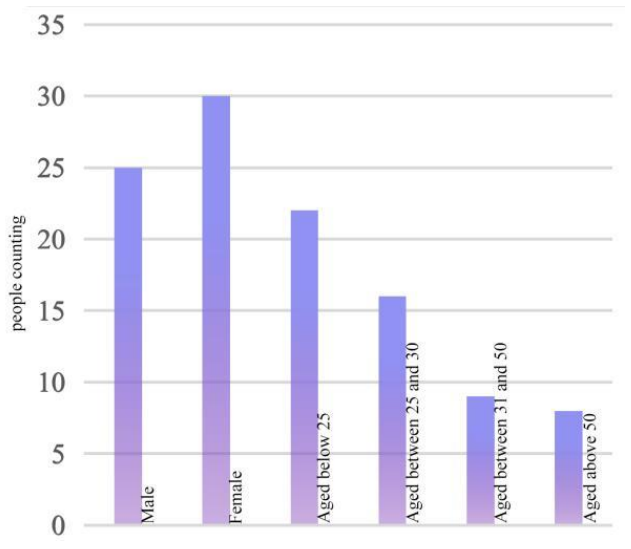

Figure 2 Composition of investigated groups (compiled by the author)

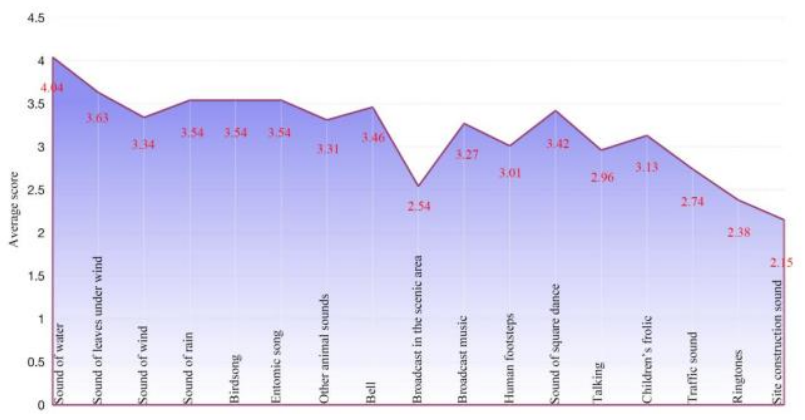

Figure 3 Survey results of sound evaluation (compiled by the author)

Table 2 Correlation analysis of scores in sound survey results

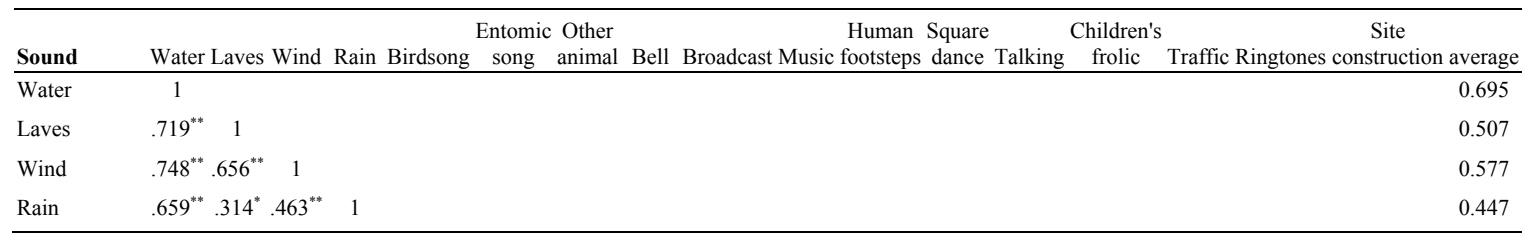




\begin{tabular}{|c|c|c|c|c|c|c|c|c|c|c|c|c|c|c|c|}
\hline Birdsong & $.641^{* *} .507^{* *} .563^{* *} .466^{* *}$ & 1 & & & & & & & & & & & & & 0.518 \\
\hline Entomic song & $.789^{* *} .654^{* *} .692^{* *} .520^{* *}$ & $.580^{* *}$ & 1 & & & & & & & & & & & & 0.559 \\
\hline Other animal & $.813^{* *} \cdot 630^{* *} \cdot 678^{* *} \cdot 519^{* *}$ & $.556^{* *}$ & $.655^{* *}$ & 1 & & & & & & & & & & & 0.586 \\
\hline Bell & $.621^{* *} .533^{* *} .595^{* *} .485^{* *}$ & $.426^{* *}$ & $.479^{* *}$ & $.483^{* *}$ & 1 & & & & & & & & & & 0.466 \\
\hline Broadcast & $.588^{* *} .443^{* *} .477^{* *} 0.176$ & $.337^{*}$ & $.305^{*}$ & $.581^{* *}$ & $.321^{*}$ & 1 & & & & & & & & & 0.453 \\
\hline Music & $.745^{* *} .435^{* *} .580^{* *} .452^{* *}$ & $.514^{* * *}$ & $.536^{* *}$ & $.517^{* *}$ & $.519^{* * *}$ & $.538^{* *}$ & 1 & & & & & & & & 0.567 \\
\hline $\begin{array}{l}\text { Human } \\
\text { footsteps }\end{array}$ & $.683^{* * *} .524^{* *} .453^{* *} .291^{*}$ & $.640^{* *}$ & $.513^{* *}$ & $.532^{* *}$ & $.351^{* *}$ & $.540^{* *}$ & $.616^{* *}$ & 1 & & & & & & & 0.522 \\
\hline Square dance & $.626^{* *} .415^{* *} .480^{* *} .361^{* *}$ & $.416^{* *}$ & $.425^{* *}$ & $.466^{* *}$ & $.436^{* *}$ & $.441^{* *}$ & $.567^{* *}$ & $.425^{* *}$ & 1 & & & & & & 0.49 \\
\hline Talking & $.567^{* *} .432^{* *} .446^{* *} .488^{* *}$ & $.297^{*}$ & $.491^{* *}$ & $.476^{* *}$ & $.385^{* *}$ & $.320^{*}$ & $.486^{* *}$ & $.435^{* *}$ & $.553^{* *}$ & 1 & & & & & 0.457 \\
\hline $\begin{array}{l}\text { Children's } \\
\text { frolic }\end{array}$ & $.782^{* *} .502^{* *} .618^{* *} .511^{* *}$ & $.642^{* *}$ & $.665^{* *}$ & $.706^{* *}$ & $.463^{* *}$ & $.566^{* *}$ & $.626^{* *}$ & $.651^{* *}$ & $.584^{* *}$ & $.452^{* *}$ & 1 & & & & 0.61 \\
\hline Traffic & $.687^{* *} .325^{*} .544^{* *} .466^{* *}$ & $.592^{* *}$ & $.457^{* *}$ & $.602^{* *}$ & $.338^{*}$ & $.582^{* *}$ & $.708^{* *}$ & $.629^{* *}$ & $.526^{* * *}$ & $.454^{* *}$ & $.645^{* *}$ & 1 & & & 0.547 \\
\hline Ringtones & $.643^{* *} .497^{* *} .570^{* *} .440^{* *}$ & $.632^{* *}$ & $.562^{* *}$ & $.586^{* *}$ & $.497^{* *}$ & $.480^{* *}$ & $.542^{* *}$ & $.524^{* *}$ & $.497^{* *}$ & $.410^{* *}$ & $.631^{* *}$ & $.627^{* *}$ & 1 & & 0.546 \\
\hline $\begin{array}{l}\text { Site } \\
\text { construction }\end{array}$ & $.809^{* * *} .519^{* *} .672^{* *} .543^{* *}$ & $.493^{* *}$ & $.617^{* *}$ & $.580^{* *}$ & $.530^{* *}$ & $.546^{* *}$ & $.717^{* *}$ & $.551^{* *}$ & $.620^{* * *}$ & $.622^{* *}$ & $.709^{* * *}$ & $.577^{* *}$ & $.604^{* * *}$ & 1 & 0.607 \\
\hline
\end{tabular}

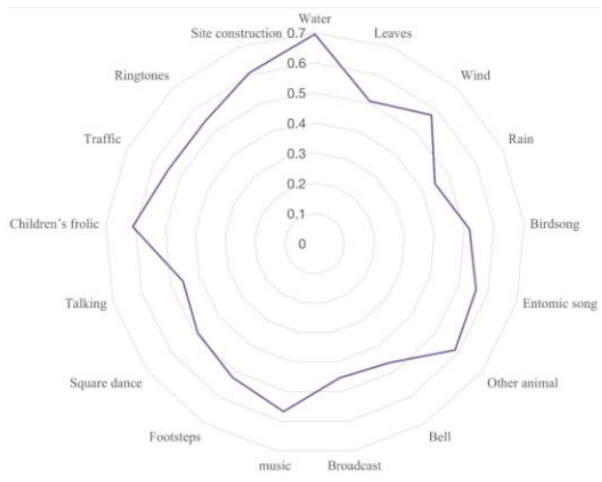

Figure 4 Correlation average of sound scores (compiled by the author)

\subsection{Schematic design stage}

\subsubsection{Sound design}

The specific procedure of sound design should be planned based on the sound structure of "keynote sounds - signal sounds - sign sounds" mentioned above and realize the effective transition from the initial acoustic environment to the feedback acoustic environment by retaining the sound with positive evaluation results, removing noise and incorporating reasonable artificial sounds. According to the survey results, the author forms a natural soundscape effect dominated by the sound of flowing water and wind by using the original sound of water and wind in the place as keynote sounds, incorporating the "sound of dripping water" in the way of artificial sound as the signal sound for the contact between people and the interactive device, and enhancing the sound of flowing water as the sign sound for the final output of the device.

\subsubsection{Interactive device design}

The application of tools and algorithm simulation at the control terminal, the output mode of the output terminal and hardware implementation patterns are important content of interactive device design. In this example, the graphical programming language Processing is used as a design tool. It intends to form a natural acoustic environment effect of motion in silence with sound input and audiovisual output as the main interaction mode and the combination of the visual output effect of "ocean current pattern" and the sound of flowing water and leaves in the place. The pattern output changes in real time with the volume of human voice. At the input terminal, the "import processing.sound.*" sound bank is imported and the sound input command and amplitude analysis command are declared so as to control the output result through sound input. At the control terminal, the amplitude analysis command algorithm "ampl.analyze" is declared to achieve control links of input and output, and "float $\mathrm{v}=$ ampl.analyze" is input to make the acceptable sound amplitude of the device at a normal level of sound that humans can receive and make. At the output terminal, the projection on the ground is considered as the output of visual effect (Table 4).

Table 3 Some code settings of Processing sound interaction commands

(Revised according to the summary of literature ${ }^{[11]}$ )

\begin{tabular}{|c|c|}
\hline $\begin{array}{l}\text { Input code setting of the main } \\
\text { program }\end{array}$ & $\begin{array}{l}\text { Graphic calculation code setting } \\
\text { for amplitude analysis }\end{array}$ \\
\hline import & void draw() \\
\hline processing.sound.*;//Import & frameRate $(20) ;)$; \\
\hline the sound bank & float $\mathrm{v}=$ ampl.analyze ()$; / /$ Input \\
\hline AudioIn ai;//Declare the & the sound amplitude analysis \\
\hline microphone input command & command \\
\hline Amplitude ampl;//Declare the & alpha=v;//The alpha value \\
\hline amplitude analysis command & depends on the volume of the \\
\hline $\begin{array}{l}\text { vold setup() } \\
\{\operatorname{size}(369,369) ; / / \text { Create the }\end{array}$ & $\begin{array}{l}\text { sound input via the microphone. } \\
\text { fill }(0,255 \text {-alpha*800);//The alpha }\end{array}$ \\
\hline canvas size, for example, & value determines the refresh rate \\
\hline $369 * 369$ & of the pattern \\
\hline ai $=$ new AudioIn(this, 0$) ; / /$ Set & $\operatorname{rect}(0,0$, width,height $) ; / /$ The \\
\hline microphone input & refresh effect of the pattern is \\
\hline ai.play();//Microphone play & within the range of the canvas \\
\hline ampl = new & loadPixels();//Read pixels \\
\hline Amplitude(this);//Set the & for(Particle p:particles $)\{/ /$ cover \\
\hline amplitude & all pattern particles \\
\hline ampl.input(ai);//Amplitude & p.move ()$;\}$ \\
\hline input $\}$ & updatePixels();//Update pixels $\}$ \\
\hline
\end{tabular}




\subsubsection{Program description}

In this case, the interactive device produces visual and auditory output through the environment and human voice, thus achieving real-time interaction among sounds, the environment and listeners' voice. Under the influence of sounds in the external environment, the device presents the visual effect of slow moving dynamic "particles", forming a static and natural effect of harmony with the environment. When listeners enter the site, the "particles" gradually accelerate their motion and form an "ocean current effect" as the number of people or sounds increases. Meanwhile, the device makes the sound of dripping and flowing water immediately, forming a natural effect of motion in silence with the environment and environmental sounds (Figure 5).

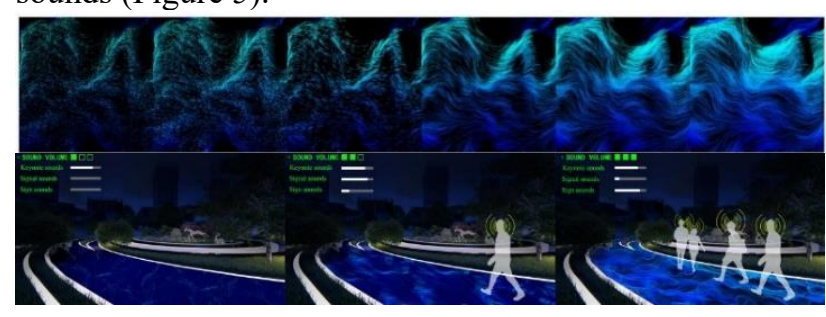

Figure 5 Sound visualization effect with particle effect as an example(For codes of particle movement, refer to https://www.openprocessing.org/sketch/854271)

\section{Conclusion}

The interactive soundscape for urban landscape is a new type of landscape in the era of the development of interconnection technology as well as an important medium for users to generate dialogues and emotional resonance with the landscape environment through sound. It should be designed based on adequate investigations on site sounds and detailed classification research on the composition of the initial soundscape at the site. It is required to make reasonable planning for the feedback acoustic environment of the design results based on the real sound experience demand of site users, combine the landscape sound with modern digital design technology and realize and operate interactive devices in a scientific way so as to build a sound landscape with better experience.

\section{References}

1. ISO. Acoustics-Soundscape-Part 1: Definition and Conceptual Framework[S]. 2014.

2. Hao Yiying, Shao Yuhan. The role of soundscape methods in landscape design $[\mathrm{J}]$. Landscape Architecture, 2020, 8(01): 72-83.

3. Luo Lixian. Research on the design of soundscape sketches in urban public open space [D]. Harbin Institute of Technology, 2010.

4. Sun Yingying. Evaluation and Construction of Urban Park Soundscape [D]. Shandong Agricultural University, 2012.

5. Yuan Xiaomei. The formation and evolution of soundscape thoughts for classical Chinese gardens $[\mathrm{J}]$. Chinese Garden, 2009, 25(07): 32-38.

6. Yan Min. Sound - Music - Scenery - A study of soundscape aesthetics of Zhuangzi based on the concept of "Sound of Nature" [J]. Huazhong Architecture, 2020, 38(06): 131-134.

7. Liu Jiang, Yu Shanshan, Wang Yajun, Zhang Jinqing. Research on the interaction between urban park landscape and soundscape experience [J]. Chinese Garden, 2017, 33(12): 86-90.

8. Ge Tianji, Zhu Xun, Ye Hechen. Research on the difference of soundscape evaluation of different birdsongs in urban parks [J]. Urban Architecture, 2018(23): 32-35.

9. LIU J, Kang J, Behm H, et al. Effects of landscape on soundscape perception: soundwalks in city parks[J]. Landscape and Urban Planning, 2014, 123: 30-40.

10. Liu Jiang, Yang Ling. A review of soundscape research of urban open space $[\mathrm{J}]$. Architecture and Culture, 2019(12): 156-158.

11. Weng Mei. Auditory landscape design [J]. Chinese Garden, 2007(12): 46-51.

12. Edited by Alan Cooper and Robert Reimann; translated by Zhan Jianfeng et al. Software concept revolution - the essence of interactive design [M]. Publishing House of Electronics Industry, 2005 (6): 210.

13. Yu Gang. Definition of interactive architecture [J]. Architecture and Culture, 2012(08): 62-63.

14. Hunter M D, Eickhoff S B, Pheasant R J, et al. The state of tranquility: Subjective perception is shaped by contextual modulation of auditory connectivity[J]. NeuroImage, 2010, 53: 611-618. 